\title{
Heart Rate-Dependent Characteristics of Diastolic Ventricular Filling in the Developing Chick Embryo
}

\author{
CYNTHIA M. PHELAN, SHARON F. HUGHES, AND D. WOODROW BENSON, JR. \\ Children's Memorial Hospital, Division of Pediatric Cardiology, Willis J. Pott's Children's Heart Center, \\ Northwestern University Medical School, and the Feinberg Cardiovascular Research Institute, \\ Chicago, Illinois 60614
}

\section{ABSTRACT}

\begin{abstract}
The contributions of the early (passive) and late (active) components of ventricular filling have been reported to decrease and increase, respectively, during chick embryo cardiac development. We hypothesized that the observed changes in ventricular filling during early cardiac development results from a decrease in cycle length. We studied the effect of development and cycle length on atrioventricular inflow in 28 chick embryos, Hamilton-Hamburger stages 17, 24, and 26. Cycle length was perturbed (range $240-1040 \mathrm{~ms}$ ) in ovo by transiently heating or cooling the sinus venosus. Atrioventricular inflow and dorsal aortic velocities were obtained by $20-\mathrm{MHz}$ pulsed Doppler flowmeter and digitally recorded at 500 samples per second. Stroke volume was calculated from dorsal aortic velocity and crosssectional area. The atrioventricular inflow wave form was inte-
\end{abstract}

The diastolic filling characteristics of the chick embryo ventricle change during developmental stages 12 through 27; the contributions of the early (passive) and late (active) components of ventricular filling have been reported to decrease and increase, respectively (1). Previous studies of altered embryonic heart rate using environmental temperature (1), cardiac pacing (2), and the thermal probe technique (3) have shown that early diastolic filling is heart rate dependent. This study further analyzes the differences in early ventricular filling velocities and flows resulting from heart rate perturbation. We found ventricular filling characteristics to be both developmentally determined and cycle length dependent.

\section{METHODS}

Twenty-eight fertile white Leghorn chicken eggs were incubated blunt end up at $38^{\circ} \mathrm{C}$ in a forced draft incubator to Hamburger-Hamilton stages $17(n=7,3 \mathrm{~d}), 23-24(n=10$,

Received March 24, 1994; accepted November 4, 1994.

Correspondence and reprint requests: D. Woodrow Benson, Jr., Division of Cardiology \#21, Children's Memorial Hospital, 2300 Children's Plaza, Chicago, IL 60614.

Supported in part by the Walden W. and Jean Young Shaw Foundation and by the Summer Sciences Research Program for Medical Students T8-FY-92-1061 from The March of Dimes Birth Defects Foundation. grated and partitioned by area and percentage of total into early (passive) and late (active) components using three methods. Regardless of method, the proportion of filling volume due to the early and late components was cycle length and stage dependent $(p<0.05)$. The early and late filling volumes were large in the older embryos, and during cycle length decrease (heart rate increase) the early filling volume decrease was greater than the late filling volume decrease. When compared with the percentage of intrinsic heart rate, the percentage of stroke volume due to early filling decreased as heart rate increased and was greater in younger embryos at all heart rates. That due to late filling increased as the percentage of intrinsic heart rate increased. Ventricular filling characteristics are both developmentally determined and cycle length dependent. (Pediatr Res 37: 289-293, 1995)

$4 \mathrm{~d})$, and $25-26(n=11,5 \mathrm{~d})(4)$. Because of the similarity between adjacent stages, stage $23(n=1)$ and stage $24(n=9)$ embryos were consolidated into one group referred to as stage $24(n=10)$. Likewise, stages $25(n=2)$ and stage $26(n=9)$ were combined into stage $26(n=11)$. Eggs were placed in a heated sand bath (Bioengineering Department, Medical College of Georgia, Augusta, GA) under radiant warmers to maintain a constant egg temperature of 37 to $38^{\circ} \mathrm{C}$. The embryo was exposed by cutting a window into the blunt end of the egg and removing extraembryonic and pericardial membranes. Any deformed embryos or embryos that bled during the procedure were discarded. Each study was performed in less than $10 \mathrm{~min}$.

Blood velocity measurements were taken with a $20-\mathrm{MHz}$ pulsed Doppler velocity meter (Bioengineering Department, University of Iowa, Iowa City, IA) that has a linear response to a maximal value of $100 \mathrm{~mm} / \mathrm{s}$. Velocity wave forms were digitally recorded at 0.002 -s intervals (500 samples per second) and analyzed by a physiorecorder (RC Electronics Computerscope, Santa Barbara, CA). While viewing the embryo through a dissecting microscope, we positioned piezoelectric crystals (diameter $0.75 \mathrm{~mm}$ ) at a $45^{\circ}$ angle to the dorsal aorta between the aortic arches and the limb bud and at the ventricular apex to obtain simultaneous blood velocity readings. The apex 
crystal was manipulated to obtain the characteristic atrioventricular inflow velocity wave form.

Dorsal aortic blood flow. The diameter (D) of the dorsal aorta was measured directly with a micrometer, and crosssectional area $\left[\pi(\mathrm{D} / 2)^{2}\right]$ was calculated. Dorsal aortic blood flow $\left(\mathrm{mm}^{3} / \mathrm{s}\right)$ was calculated as a product of phasic dorsal aortic velocity and cross-sectional area. This method does not measure blood flow to the head, which is estimated to be less than $10 \%$ of total flow (2). Stroke volume $\left(\mathrm{mm}^{3}\right)$ was determined for each cardiac cycle by dividing aortic blood flow by the heart rate, which was derived from the inverse of the previous cycle length.

Partitioning of the atrioventricular inflow velocity wave form. We defined the start of atrioventricular inflow as the point at which the dorsal aortic wave form returns to the baseline (Fig. 1). We divided the atrioventricular inflow (or phasic ventricular filling) velocity profile into two components: early (passive) filling component and late (active) filling component. The start of the early filling phase coincides with the upstroke of the atrioventricular inflow wave form and follows the dorsal aortic wave form descent to baseline. The onset of the late phase coincides with the most rapid upstroke within the atrioventricular wave form. The end of the late phase coincides with the end of ventricular filling, the point at which the wave form reaches the baseline, as well as with the upstroke of dorsal aortic wave form, which represents the outflow of that blood volume. Because the early and late components often overlap, we realized that there is some ambiguity inherent in assigning the exact start of late filling and the exact end point of early filling. We therefore used three different methods to partition the atrioventricular inflow to determine whether this ambiguity would affect our analysis of cycle length-dependent changes in diastolic filling.

Method 1 (Fig. 2A) assumed that early filling ends when late filling begins and that the force of atrial contraction supersedes the pressure gradient that drives early filling (1). Therefore, the

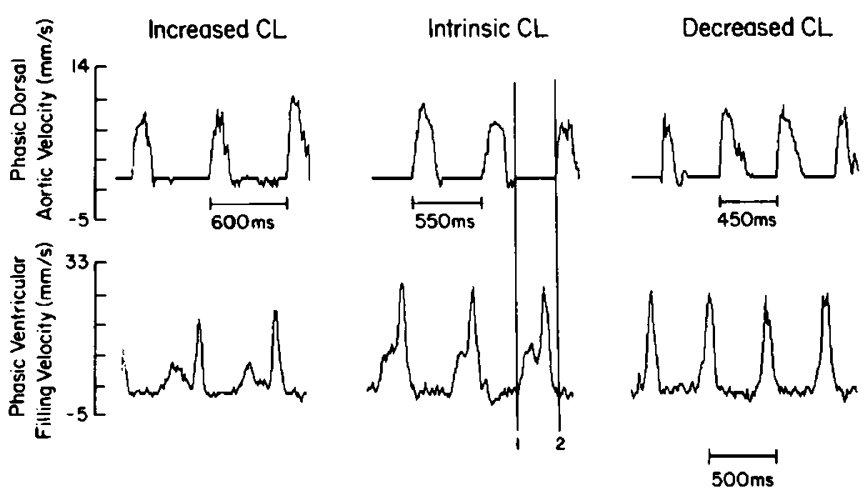

Figure 1. Cycle length $(C L)$ perturbation in a stage 24 chick embryo. The beginning and end of ventricular filling are indicated on the phasic ventricular filling velocity wave forms at intrinsic CL. Atrioventricular inflow begins when the phasic dorsal aortic velocity wave form returns to the baseline (1) and ends when the atrioventricular inflow wave form returns to baseline, coinciding with the upstroke of the next dorsal aortic wave form (2). Application of cold probe to the sinus venosus prolonged the cycle length to $600 \mathrm{~ms}$ and early and late filling components of the phasic ventricular filling velocity wave form separated. Application of hot probe shortened cycle length to $450 \mathrm{~ms}$, and the two components fused together.
A. Method I

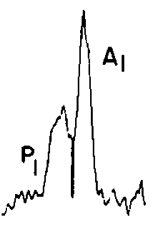

B. Method 2

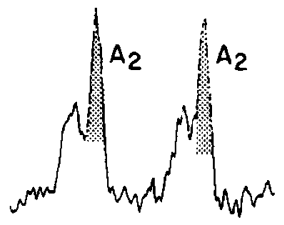

$P_{2}=$ Total $-A_{2}$

$B_{2} \cdot A_{2}=$ Constant

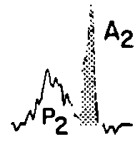

C. Method 3

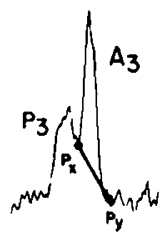

$250 \mathrm{~ms}$
Figure 2. Methods for partitioning of the early and late components of atrioventricular inflow in the chick embryo. $A$, Method 1 assumes that passive filling component $\left(P_{l}\right)$ ends when the active component $\left(A_{l}\right)$ begins and partitions the wave form with a perpendicular line. $B_{l}$, Method 2 assumes that the volume of blood propelled by atrial contraction (or during the active filling phase) is independent of changes in cycle length. A constant value for active filling was obtained from beats with long cycle length $\left(B_{2}\right)$. The passive filling component $\left(P_{2}\right)$ was calculated as the difference between total atrioventricular inflow and the active filling component $\left(A_{2}\right)$, which is treated as a constant. $C$, Method 3 assumes that passive filling continues during active filling and that the active filling cormponent wave form is superimposed on the passive component wave forrn. The method 3 passive filling component $\left(P_{3}\right)$ was estimated by extending a diagonal line from the rapid upstroke of active filling (point $P_{x}$ ) to the baseline (point $P_{y}$ ) and determining the area under that line.

early and late components were partitioned by a straight line, perpendicular to the baseline, at the point of the wave form nadir.

Method 2 (Fig. $2 B$ ) assumed that the late contribution remained constant, i.e. that the volume of blood propelled by atrial contraction is the same for all heartbeats in a given embryo, regardless of cycle length, as suggested by previous studies (3). Because at slow heart rates the two components of the atrioventricular wave form separate (Fig. $2 B$ ), we could obtain isolated late component wave forms for embryos with very slow heart rates. The area of the late filling wave form (A) was calculated for each beat and subtracted from the total area of the ventricular filling wave form to obtain values for early filling area $(p=$ total $-\mathrm{A})$.

Method 3 (Fig. 2C) assumed that early filling may continue after late filling begins. Using this method, early filling continued according to a linear interpolation that estimated the slope of the early filling to be a straight line extending from the start of the late component to the baseline when filling ended. Early filling corresponded to the area under this portion of the curve.

Cycle length perturbation. A stainless steel probe (1-mm diameter) was heated to approximately $100^{\circ} \mathrm{C}$ in a boiling water bath, or cooled to $0^{\circ} \mathrm{C}$ in an ice water bath, and placed on the sinus venosus to perturb cycle length (5). Velocity wave forms were recorded for 10-20 s after each probe application.

Analysis of data. For each embryo, we analyzed wave forms from 15 cardiac cycles: five at intrinsic cycle lengths, five at decreased cycle lengths, and five at increased cycle lengths. The atrioventricular inflow wave form was integrated (total area) and, for each of the three partitioning methods, the early 
and late components of the atrioventricular wave form were obtained. The fraction of the total atrioventricular inflow contributed by early and late components was determined as a ratio of the area of each component to the total area. These fractions were converted to percentage values and designated as the percentage contribution of the early (percentage due to passive filling) or late filling (percentage due to active filling) to the total atrioventricular inflow wave form. We assumed that for each cardiac cycle the atrioventricular inflow volume would be identical to the next stroke volume, and we determined early and late filling volume to be the product of stroke volume times the fraction due to early or late filling, respectively. The ratio of the early filling volume to the stroke volume, expressed as a percentage of stroke volume contributed by early filling, is identical to the percentage contributed to atrioventricular inflow volume by early filling. Some comparisons were made to the percentage of intrinsic heart rate, which was determined from the beat-to-beat cycle length and intrinsic cycle length. Regression analysis was performed to assess cycle length or heart rate-dependent changes. Differences between methods 1 and 3 were compared by regression analysis of the difference in the estimates of the two methods for each embryo. Differences among stage 17,24 , and 26 embryos were compared by applying a paired $t$ test to the slopes of the regression lines for each embryo.

\section{RESULTS}

Heart rate perturbation resulted in a cycle length range of 240-1040 ms (57-250 beats per minute) across all stages (Table 1). An example of the effects of heart rate perturbation on dorsal aortic and ventricular filling velocity is shown for a stage 24 embryo (Fig. 1).

Method 2 could not be used in every embryo because of difficulty in obtaining sufficiently prolonged cycle length to achieve separation of the active and passive filling wave forms (Fig. 2). We were able to perform the method 2 analysis on only six embryos-two from each stage. Because of this technical limitation, and because the physiologic assumptions are highly speculative, we performed no additional analysis of method 2.

Figure 3 illustrates the regression analyses of the percentage of stroke volume contributed by the two components of atrioventricular inflow in individual stage 17,24 , or 26 embryos. Significant cycle length dependence for both the early and late components of atrioventricular inflow was present in the embryos from each stage. The response of the early and late filling volume to changes in cycle length for all embryos in the three stages is shown in Figure 4. The early filling volume was dependent on both cycle length and developmental stage. Early filling volume was larger in older embryos; for all stages, early

Table 1. Cycle length (mean $\pm S D)$

\begin{tabular}{llccc}
\hline & $\begin{array}{c}\text { Cold probe } \\
(\mathrm{ms})\end{array}$ & $\begin{array}{c}\text { Intrinsic } \\
(\mathrm{ms})\end{array}$ & $\begin{array}{c}\text { Hot probe } \\
(\mathrm{ms})\end{array}$ & $\begin{array}{c}\text { Range } \\
(\mathrm{ms})\end{array}$ \\
\hline Stage 17 & $705 \pm 151$ & $507 \pm 44$ & $415 \pm 56$ & $300-1040$ \\
Stage 24 & $580 \pm 56$ & $446 \pm 56$ & $337 \pm 52$ & $240-740$ \\
Stage 26 & $564 \pm 117$ & $395 \pm 29$ & $336 \pm 34$ & $270-710$ \\
\hline
\end{tabular}
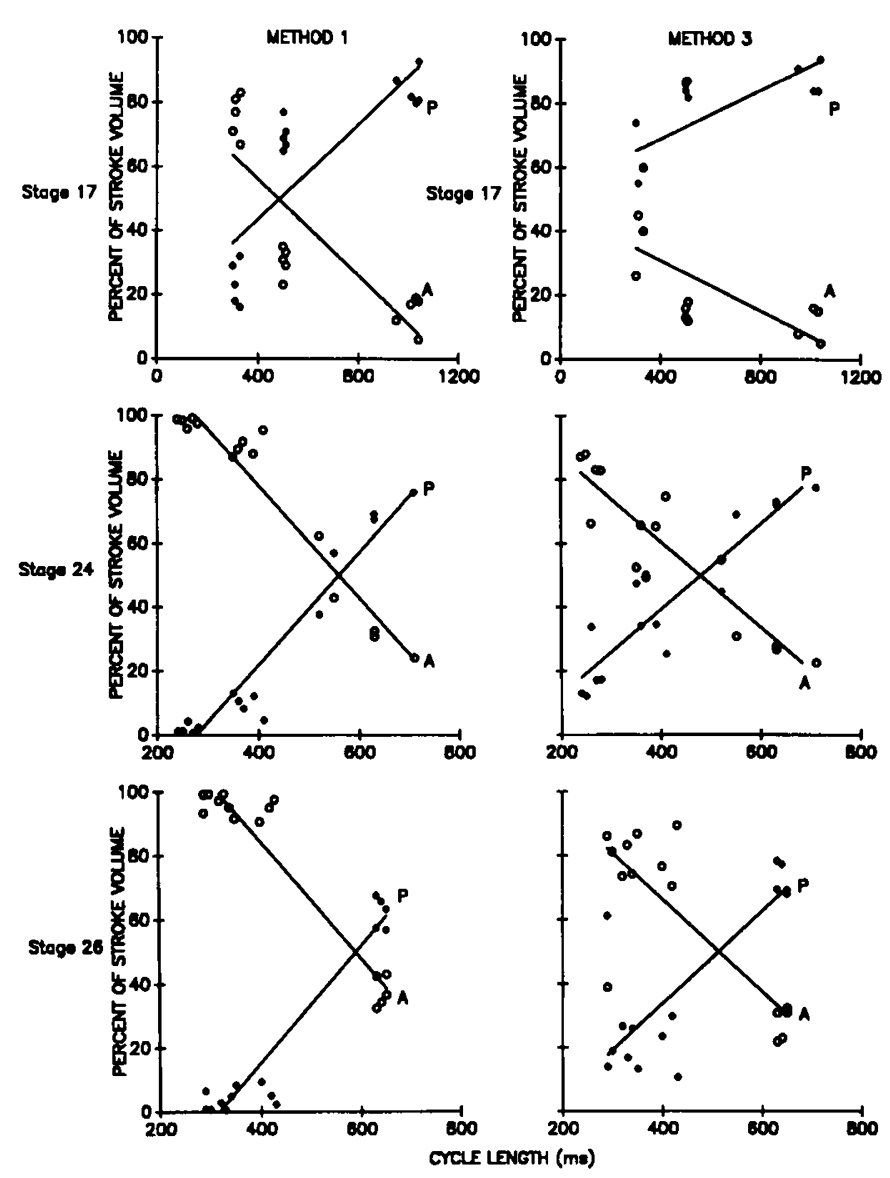

Figure 3. Comparison of methods 1 and 3 for individual stage 17, 24, and 26 embryos. Lines of regression of percentage of stroke volume contributed by early filling $(P, O)$ and late filling $(A, O)$ as a function of cycle length are illustrated. Regression analyses for the early and late components of method 1 and method 3 showed significant cycle length-dependent relationships for the early and late components for all three embryos.

filling volume increased as cycle length increased (heart rate decreased). Method 1 gave a consistently smaller early filling volume and larger late filling volume than did method 3 ( $p<$ 0.05 ). The late filling volume was larger in older embryos, and for all embryos, the late filling volume decreased as cycle length increased (heart rate decreased).

To investigate the possibility that the stage dependence of early filling was not merely due to the well-known stage and heart rate (cycle length) dependence of stroke volume $(2,6)$, we evaluated the relationship between the percentage of stroke volume due to early filling or late filling compared with the percentage of intrinsic heart rate as illustrated in Figure 5. All stages showed a decrease in percentage of stroke volume due to early filling as the percentage of intrinsic heart rate increased: The percentage of stroke volume was greater for younger embryos for a given percentage heart rate than for older embryos. In contrast, the percentage of strike volume due to late filling increased as the percentage of intrinsic heart rate increased and was greater in the older embryos.

\section{DISCUSSION}

This study confirms that diastolic ventricular filling characteristics are developmentally determined and characterizes 

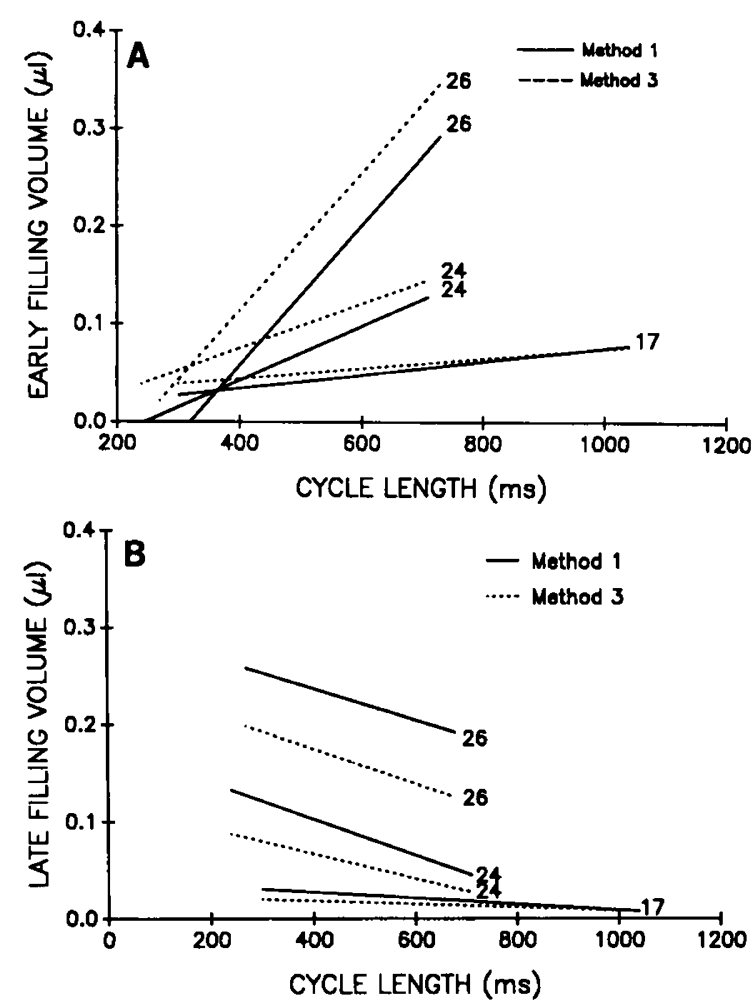

Figure 4. Comparison of two atrioventricular inflow partitioning methods. Lines of regression were obtained from all embryos at each stage to illustrate the relationship between cycle length and filling volume during the early filling phase $(A)$ and late filling volume $(B)$ as determined by method 1 (solid line) and method 3 (dashed line). For both methods, the relationship was both cycle length and stage dependent. The stage 17 chicks were capable of more extensive cycle length increases with cooling.

their cycle length dependence. Regardless of the method of analysis, the relative contribution of the early (passive) and late (active) components of ventricular filling are dependent on cycle length. When the filling volumes are normalized by stroke volume at intrinsic cycle length, cycle length dependence remains, and significant differences between the filling characteristics of younger and older embryos are evident.

We examined different methods of partitioning the passive and active components of the atrioventricular inflow wave form. Method 2, which assumes a constant contribution from the active (late) component, proved to be an unsatisfactory partitioning method. In addition to the technical difficulty in using this approach, it may use physiologically unreasonable assumptions. Methods 1 and 3 produced qualitatively similar results. Based on the similarity of results between methods 1 and 3 and the relatively simplicity of application, we recommend using method 1 .

The cycle length dependence of ventricular filling characteristics has been recognized previously. Hu et al. (1) attempted to control for cycle length-dependent effects by selecting embryos with similar heart rates. The similarity of heart rate may have resulted from embryo temperature change during study; this is important because hypo- and hyperthermia affect cardiac output, arterial pressure, and afterload and thereby may affect filling characteristics $(7,8)$. By using the thermal probe technique (3), isolated heart rate effects could be studied. We found that the early filling volume decreased and
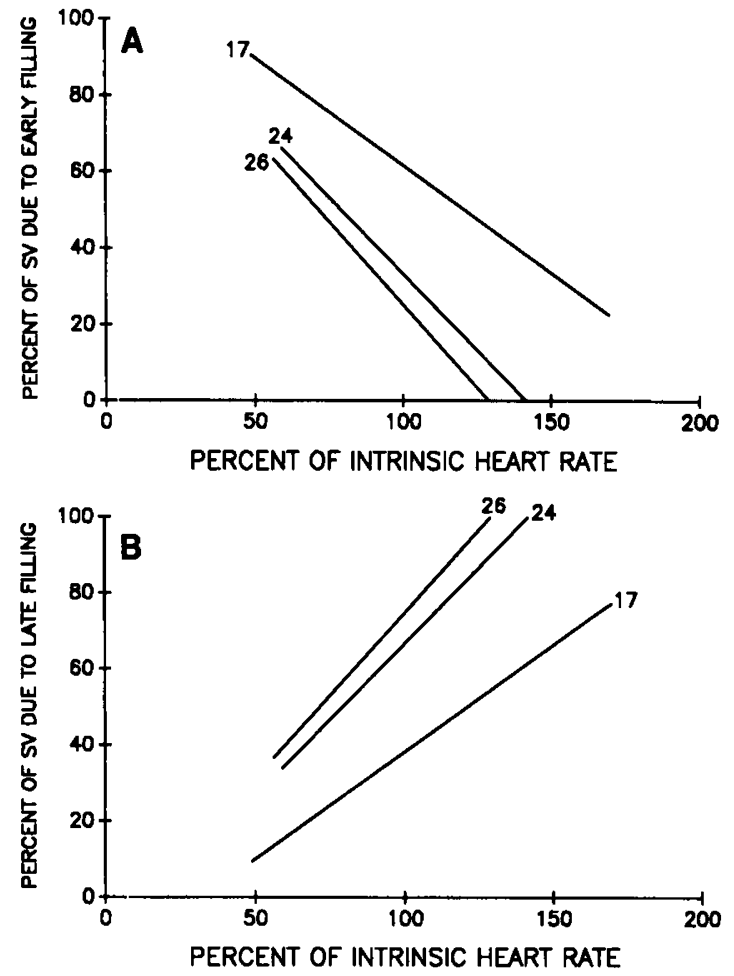

Figure 5. Comparison of the percentage of stroke volume (SV) due to the early (passive) and late (active) components of ventricular inflow to percentage of intrinsic heart rate for all embryos at each stage. Partitioning was performed using method 1 . In stage 17 embryos, the percentage of stroke volume due to early filling $(A)$ is greater and the percentage due to late filling $(B)$ is less at all intrinsic heart rates compared with the older embryos.

the late filling volume increased as cycle length decreased. However, when the known stage (developmental) and heart rate dependence of stroke volume (and filling volume) were taken into account, a reciprocal relationship persisted: The percentage of stroke volume due to early filling decreased and the percentage due to late filling increased as the percentage of intrinsic heart rate increased.

To study the developmental changes in ventricular filling characteristics, it is necessary to account for the both developmental increase in stroke volume and decrease in cycle length during early chick embryo cardiac development $(2,6,9)$. Because stroke volume is linearly related to end diastolic ventricular dimension (10), we evaluated stage-dependent filling characteristics by normalizing the diastolic filling volumes by stroke volume. We found stage-dependent differences in filling characteristics. The percentage of ventricular filling due to early atrioventricular inflow decreases during development of the preinnervated chick embryo heart, whereas the actual early volume increases in a manner similar to the developmental increase in stroke volume. As shown in this study, the percentage of stroke volume due to early ventricular filling is increased and the percentage due to late filling is decreased at all percentages of intrinsic heart rates in younger versus older stages of development. We do not know whether this is due to developmental changes in ventricular compliance (pressurevolume relationship), ventricular relaxation, or venoatrial versus atrioventricular coupling. 
Diastolic filling is determined by the interaction of the active and passive relaxation of the ventricle and the force exerted on the noncontracting ventricle by the venoatrial preload system. It is becoming increasingly clear that ventricular suction plays a role in ventricular filling (11-13). Hu et al. (1) associated the developmentally dependent shift from predominately passive to active filling characteristics with the appearance of trabeculations and inferred a decrease in ventricular compliance. Results of the present study support this idea. Additional studies are necessary to define the physiologic determinants of venoatrial and atrioventricular coupling.

\section{REFERENCES}

1. Hu N, Connuck DM, Keller BB, Clark EB 1991 Diastolic filling characteristics in the stage 12 to 27 chick embryo ventricle. Pediatr Res 29:334-337

2. Hu N, Clark EB 1989 Hemodynamics of the stage 12 to 29 chick embryo. Circ Res $65: 1665-1670$

3. Dunnigan A, Hu N, Benson DW Jr, Clark EB 1987 Effect of heart rate increase on dorsal aortic blood flow in the chick embryo, stages 18-24. Pediatr Res 17:945-948
4. Hamburger V, Hamilton HL 1951 A series of normal stages in the development of the chick embryo. J Morphol 88:49-92

5. Cuneo B, Hughes S, Benson DW 1991 Heart rate perturbation in chick embryos: a comparison of two methods. Am J Physiol 260:H1864-H1869

6. Cuneo B, Hughes S, Benson DW 1993 Heart rate perturbation in the stage 17:27 chick embryo: effect on stroke volume and aortic flow. Am J Physiol 264:H755-H759

7. Nakazawa M, Miyagawa S, Takao A, Clark EB, Hu N 1986 Hemodynamic effects of environmental hyperthermia in stage 18, 21, and 24 embryos. Pediatr Res 20:12131215

8. Nakazawa M, Clark EB, Hu N, Wispe J 1985 Effect of environmental hypothermia on vitelline artery blood pressure and vascular resistance in the stage 18, 21 and 24 chick embryos. Pediatr Res 19:651-654

9. Clark EB, Hu N 1982 Developmental hemodynamic changes in the chick embryo stages 18 to 27 . Circ Res $51: 810-815$

10. Keller BB, Hu N, Clark EB 1990 Correlation of ventricular area, perimeter, and conotruncal diameter with ventricular mass and function in the chick embryo from stage 12 to 24 . Circ Res 66:109-114

11. Taber LA, Hu N, Pexieder T, Clark EB, Keller BB 1993 Residual strain in the ventricle of the stage 16-24 chick embryo. Circ Res 72:455-462

12. Keller BB, Tinney JP, Hu N 1994 Embryonic ventricular diastolic and systolic pressure-volume relations. Cardiol Young 4:19-27

13. Braunstein JB, Donovan M, Hughes S, Benson DW Jr 1994 Assessment of ventricular relaxation in the developing chick embryo using a monoexponential model. Am J Physiol 267:H631-H635 